\title{
Use of Bi-plane Mode of Transesophageal Echocardiography for Real Time Visualization and Cannulation of the Internal Jugular Vein: A Safe and effective Alternative
}

\author{
Harshil Joshi', Vijaya Kumara', Guruprasad Rai ${ }^{1}$ \\ ${ }^{1}$ Assistant Professor, Manipal Academy of Higher Education, Kasturba Medical College, Manipal.
}

\section{Abstract}

Background: To check feasibility and efficacy of biplane mode of the transesophageal echocardiography (TEE) probe for use of visualization and cannulation of internal jugular vein (IJV). Subjects and Methods: This is Prospective, randomized, controlled pilot study done at tertiary care hospital. Fifty adult cardiac surgery patients in current study received right IJV catheterization using the TEE probe. Another fifty patient undergone same IJV cannulation with use of linear probe. The puncture time, success rate in first attempt, quality of the imaging with needle tip positioning and wire positioning were recorded. The occurrence of complication or any adverse event was also observed. Results: There was statistical difference in puncture time between two groups. Puncture time was less in TEE group. One failed puncture attempt with linear probe was there. There was no statistical difference in image quality with needle tip positioning and wire positioning between groups. No complications or adverse events were observed in either group. Conclusion: The Bi-plane mode of TEE probe can be used for visualization and cannulation of IJV to achieve higher success rate and decrease chance of complication with favorable feasibility and safety.

Keywords: Internal Jugular Vein, Ultrasound, Transesophageal Echocardiography, Cardiac surgery.

Corresponding Author: Dr. Harshil Joshi, Assistant Professor,Department of Anesthesia, Manipal Academy of Higher Education, Kasturba Medical College, Manipal.

Received: September 2019

Accepted: September 2019

\section{Introduction}

For Cardiac anesthesiologist insertion of central venous cannulation is most common procedure and right internal jugular vein (IJV) is most commonly used for the same. American Society of Echocardiography and the Society of Cardiovascular Anesthesiologists has recommended to use real-time ultrasound during IJV cannulation whenever possible. This recommendation is based on category A, level 1 evidence. $^{[1]}$ Unfortunately, risk of complication is not eliminated with ultrasound technique also. ${ }^{[2]}$ Majority of available ultrasound machine uses beam of $1 \mathrm{~mm}$ width in single plane forming 2 dimensional image. We can put transducer either perpendicular to vessel for short axis view or longitudinal for long axis view, but can't see both simultaneously. Mostly ultrasound guided IJV cannulation is done in short axis view and one major disadvantage of that is we can't see needle tip which can lead to in advert arterial puncture or posterior wall of vein. So if we can see both short and long axis simultaneously, we can null pitfalls of each other. Working Principle behind the linear ultrasound probe and phase array echocardiography is same. But transducer in echocardiography is phased array to monitor heart function while in other conventional ultrasound it is linear array. As TEE probe is multiplane and can show two perpendicular plane simultaneously, it gives better 3 dimensional virtual image of heart. Now a days due to high recommendation of TEE in cardiac surgery, most of the cardiac unit are equipped with TEE probe but not necessarily equipped with linear vascular probe. Even if it is available in hospital, it will be preoccupied for giving regional anesthesia in other surgeries

Y. Teng et al. has compared feasibility of TEE probe as surface probe for IJV cannulation but he has not used full potential of TEE probe. ${ }^{[3]}$ Here authors designed this study to investigate how biplane mode of TEE probe can give added safety and efficacy to IJV cannulation.

\section{Subjects and Methods}

This prospective, double arm, randomized pilot study was approved by institutional ethical committee. All the participant was informed about the study and written informed consent was taken from participant. Considering number of annual cardiac surgery done at author's hospital, sample size of 109 is calculated. 109 patient were recruited, between 20 to 60 years age, who were undergoing cardiac surgery with IJV cannulation. Patient with any clinical contraindication for IJV cannulation were excluded from study. Computer based program is used for randomization. 
Patients were divided in to two group, group L, linear probe was used for IJV cannulation and Group E, TEE probe is used for IJV cannulation.

After coming to OR, all patients radial artery cannulated under local anesthesia. A standard protocol for anesthesia induction and endotracheal intubation done. After that, Trendelenburg position given to the patient and PEEP of 5 $\mathrm{cm}$ of $\mathrm{H} 2 \mathrm{O}$ was given. Then routine sterilization and draping for IJV cannulation site done. The anesthesiologist put on sterile scrubs and put the probe in sterile endoscopic sleeve. Transducer will be covered under sterile plastic slip. In Group L, Linear Probe is used for IJV cannulation. Depth setting was kept at $4 \mathrm{~cm}$ and gain was adjusted to gain proper image. Anesthesiologist identified the artery and vein with shape, pulsation and Doppler. Short axis of IJV was used for Cannulation of IJV and later position of needle was confirmed by long axis view. In Group E, TEE probe was set at a depth of $4 \mathrm{~cm}$ with seek angle of 90 degree and $7 \mathrm{MHz}$ frequency. The probe was held with left hand and placed on neck between two heads of sternocleidomastoid triangle. After that anesthesiologist placed probe so that IJV came into the center of the field and then activated the biplane mode. Biplane mode showed both short axis and long axis of IJV. Some Side movements were made until the vein could be seen simultaneously in the short-axis and long axis view with the vein under the midpoint of the transducer. Depth and gain were adjusted to optimize picture quality. After that right hand was used to insert needle under the probe aiming at IJV until blood come in aspiration syringe. With biplane both short and long axis can be seen simultaneously to confirm needle and guidewire placement in IJV.

In current study, anesthesiologists having more than 5 years of experience in cardiac anesthesia were involved. All are excellent in either landmark guided or USG guided central venous cannulation. All cannulation done by experienced anesthesiologists and author was observer for procedures and doing scoring of procedure. Observer remain same for all procedure. We have recorded puncture time, success in first attempt, image quality with needle, and confirmation of wire position in all 100 patients.
Puncture time: the time from the beginning of locating IJV to the successful puncture.

1. Image Quality with needle tip during procedure: scoring

2. Blurred image with an unrecognizable IJV.

3. Blurred image with a recognizable IJV and artery in both short and long axis.

4. Clear and detectable IJV and artery but needle was visible in only one plane.

5. Clear and detectable IJV and artery with needle was visible in both short axis and long axis.

\section{Positioning of guide wire: scoring}

1. Wire is invisible or blurry

2. Wire is visible in only one plane

3. Wire is visible in both plane.

We defined procedural success as successful cannulation of IJV in first attempt as primary end point. Secondary end point measures included puncture time, quality of the image with needle tip in situ, confirmation of wire position and complications.

\section{Results}

109 cardiac surgery patients who were scheduled to receive IJV catheterization were recruited. 5 patients were excluded and 4 patients refused to participate in the study. Thus, 100 patients were analyzed (50 with linear and 50 with TEE probe) with student $\mathrm{T}$ test and chi square test using SPSS software. Age, sex, height, and weight were comparable in both groups of patients $(p>0.05)$. In Linear group one first attempt failed making the success rate of first attempts 49/50 $(98 \%)$, while the success in first attempts in the TEE group was $50 / 50(100 \%)$. There is also statistical significant difference in puncture time between both groups with $p$ value of 0.006 having less time in TEE group(Table 1). Otherwise no statistical differences were found in the image quality with needle tip positioning and wire positioning between the two groups. All the $\mathrm{p}$ values were greater than 0.05 [Table 1]. No complications or adverse events were observed between the two groups.

Table 1: Comparison of Heart rate (bpm) in two groups of patients studied.

\begin{tabular}{|c|c|c|c|c|}
\hline & 1st Anesthesiologist $(n=25)$ & 2nd Anesthesiologist ( $n=25)$ & $\mathbf{t} / \chi^{2}$ & P Value \\
\hline Age & $57 \pm 7.26$ & $57.72 \pm 7.32$ & 0.493 & 0.311 \\
\hline $\operatorname{Sex}(\mathrm{M} / \mathrm{F})$ & $38 / 12$ & $39 / 11$ & 0.0565 & 0.812 \\
\hline Weight $(\mathrm{Kg})$ & $63.48 \pm 11.26$ & $61.64 \pm 8.17$ & 0.934 & 0.176 \\
\hline Height $(\mathrm{cm})$ & $161.72 \pm 9.73$ & $159.96 \pm 7.58$ & 1.008 & 0.158 \\
\hline Puncture Time (second) & $29.24 \pm 4.53$ & $27.04 \pm 4.21$ & 2.513 & 0.006 \\
\hline Image Score $(3 / 4)$ & $25 / 25$ & $17 / 33$ & 2.627 & 0.105 \\
\hline Guide Wire Score $(2 / 3)$ & $23 / 27$ & $17 / 33$ & 1.5 & 0.22 \\
\hline
\end{tabular}

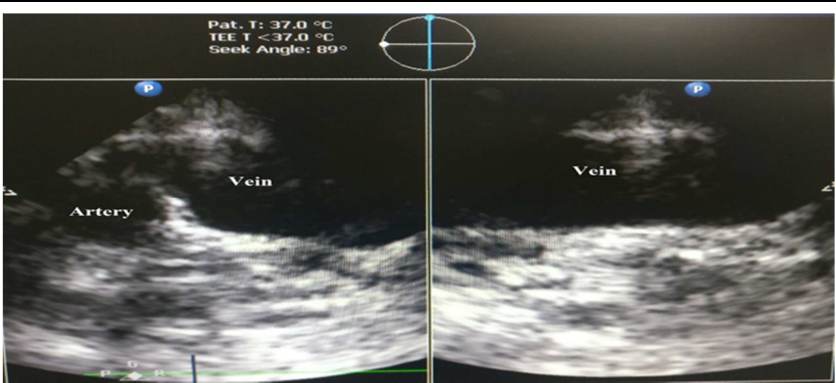

Figure 1: Bi-plane mode Visualization of Internal Jugular Vein: Short and long Axis

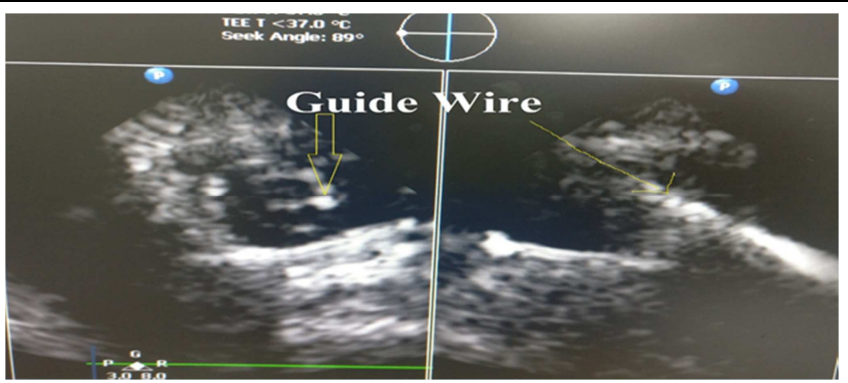

Figure 2: Bi-plane Mode Visualization of Guide-wire in IJV: In plane and Out plane 


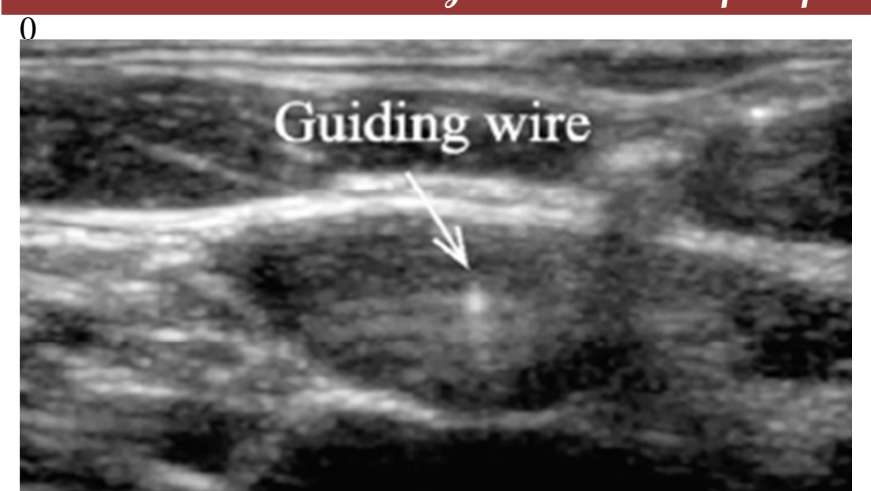

Figure 3: Short Axis of IJV with linear probe

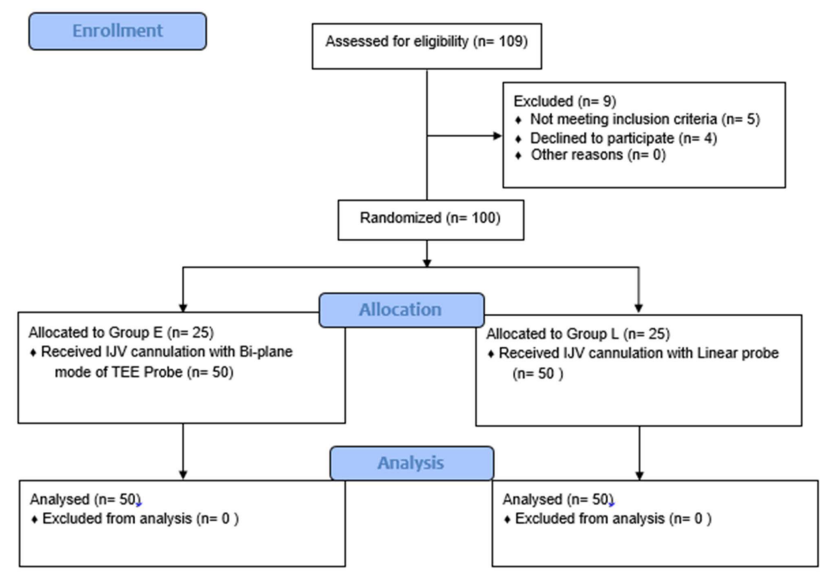

Figure 4: CONSORT Flow Diagram

\section{Discussion}

As far as author knows, No previous study has been done to see the efficacy and feasibility of simultaneous short and long axis view of IJV for cannulation in cardiac surgery. There are some case reports published. ${ }^{[4]}$ Result showed that there is $100 \%$ success rate in achieving primary end point in TEE group but success rate is $98 \%$ in group L. No significant difference was found in image quality with needle tip and guidewire visibility. No complication or adverse event were observed. Current study showed there is $100 \%$ success rate in cannulating IJV using biplane mode of TEE.

Real time ultrasonography (RTUS) is helpful to decrease complication like cannulation failure, arterial puncture and hemothorax in adult patient undergoing IJV cannulation. ${ }^{[5,6]}$ But due to inability to identify tip of the needle with certainity, serious complication has been reported with RTUS guided cannulation also. ${ }^{[7,8]}$ This is because for RTUS, we use vascular probe which is linear array probe that will show either short axis or long axis at a time. During short axis view, artery, vein and surrounding structure will be seen simultaneously but needle will be seen as pin point only or as tissue movement [Figure 3]. Occasionally, needle will pass across ultrasound beam and hit posterior wall of vein or adjacent structure because needle pathway and insertion depth is not seen in short axis view. Here long axis view will come to help, as it gives better visualization of needle course and depth so that we can avoid insertion of needle beyond target vessel. So by viewing single plane we are doing 3D task using 2D display. Needle tracking is used to overcome this limitation, but still it is not full proof.

Experiment has been done using two linear probe array positioned at 90 degree angle to each other to get both views simultaneously. ${ }^{[9]}$ Multiplane TEE is phase array probe which is very much helpful for overcoming this pitfalls. TEE probe used as surface probe for IJV cannulation will give simultaneous and real time both short and long axis image of vascular structure in single screen with activation of biplane mode [Figure 1]. So it will give advantage of both plane and will alleviate pitfalls of both. This may be the reason why puncture time is less in TEE group. In linear group, time may be consumed some time to see both short and long axis before taking puncture for evaluation of vein anatomy and surrounding structure. Yi teng et al also reported 100\% success rate for central line cannulation using TEE as surface probe and there is not much difference between vascular probe and TEE probe as per image quality. ${ }^{[3]}$ Visualization of guidewire inside vein is also very much helpful before use of dilator to prevent any consequences of complication. ${ }^{[10]}$ With this biplane mode, guidewire can be seen inside the vein without lifting or changing the probe direction [Figure 2].

Probe used in current study is 3D, but needle visualization in soft tissue is difficult with 3D. Needle and guide wire very well seen inside the vein using 3D. There were some limitation of current study. Author don't have biplane mode in Transthoracic Echocardiography probe, otherwise it was the first choice to use. Use of TEE probe as surface probe is sometime difficult, but as anesthesiologist are very much experienced they overcome this difficulty. We need to do further studies involving inexperienced and in-training anesthesiologist using bi-plane mode for evaluation of success rate and complication.

\section{Conclusion}

The luxury to watch a needle advancing in 3 planes simultaneously is an ideal development. The added information provided by the biplane views have the potential to enhance safety and accuracy during IJV cannulation and catheter placement. At present, the technique is technically more difficult than the standard 2D linear ultrasound technique. Limitations include large transducer size and increased time to learn and perform the procedure. Further studies must be planned to formally evaluate the benefits of real-time biplane ultrasonography for central venous cannulation in achieving higher success rate and decrease complications.

\section{References}

1. Christopher A. Troianos, MD, Gregg S. Hartman, MD, Kathryn E. Glas, MD, MBA, FASE, Nikolaos J. Skubas, MD, FASE, Robert T et al. Guidelines for Performing Ultrasound Guided Vascular Cannulation: Recommendations of the American Society of Echocardiography and the Society of Cardiovascular Anesthesiologists. Journal of the American Society of Echocardiography 2011;24:1291-318. DOI: 10.1213/ANE.0b013e3182407cd8

2. Hind D, Calvert N, McWilliams R, et al. Ultrasonic locating devices for central venous cannulation: metaanalysis. British Medical Journal 2003; 327: 361-7. DOI: https://doi.org/10.1136/bmj.327.7411.361 
3. Y. Teng et al. Feasibility of the Use of Transesophageal Echocardiography as a Surface Probe for Puncturing and Catheterization of the Internal Jugular Vein: A Randomized Controlled Pilot Study. Journal of Cardiothoracic and Vascular Anesthesia 32 (2018) 363-369. DOI: https://doi.org/10.1053/j.jvca.2017.10.002

4. BM, BS, Hatem A. Jlala, MBBCh, PhD et al. Real-Time ThreeDimensional Ultrasound-Guided Central Venous Catheter Placement Myles Dowling. Anesth Analg 2011;112:378 -81. DOI: 10.1213/ANE.0b013e31820521f9

5. Rothschild J, Shojania KG, Duncan BW, McDonald KM, et al. Making health care safer: a critical analysis of patient safety practices [AHRQ Evidence Reports no. 43] Association for Healthcare Research and Quality (AHRQ); 2001. AHRQ Publication 01-E058

6. Rupp SM, Apfelbaum JL, Blitt C, et al. Practice guidelines for central venous access: a report by the American Society of Anesthesiologists
Task Force on Central Venous Access. Anesthesiology 2012; 116: 53973. DOI: 10.1097/ALN.0b013e31823c9569

7. Parsons AJ and Alfa J. Carotid dissection: a complication of internal jugular vein cannulation with the use of ultrasound. Anesth Analg 2009; 109: 135-6. DOI: 10.1213/ane.0b013e3181a7f5a4

8. Stone MB and Hern HG. Inadvertent carotid artery cannulation during ultrasound guided central venous catheterization. Ann Emerg Med 2007; 49: 720. DOI: 10.1016/j.annemergmed.2006.11.030

9. Jeremy Kaplowitz and Paul Bigeleisen, "A New Biplane Ultrasound Probe for Real-Time Visualization and Cannulation of the Internal Jugular Vein," Case Reports in Anesthesiology, vol. 2014, Article ID 349797, 3 pages, 2014. https://doi.org/10.1155/2014/349797.

10. Blaivas M. Video analysis of accidental arterial cannulation with dynamic ultrasound guidance for central venous access. J Ultrasound Med 2009; 28: 1239-44. https://doi.org/10.7863/jum.2009.28.9.1239

Copyright: (ㅇ the author(s), publisher. Academia Anesthesiologica International is an Official Publication of "Society for Health Care \& Research Development". It is an open-access article distributed under the terms of the Creative Commons Attribution Non-Commercial License, which permits unrestricted non-commercial use, distribution, and reproduction in any medium, provided the original work is properly cited.

How to cite this article: Joshi H, Kumara V, Rai G. Use of Bi-plane Mode of Transesophageal Echocardiography for Real Time Visualization and Cannulation of the Internal Jugular Vein: A Safe and effective Alternative. Acad. Anesthesiol. Int. 2019;4(2):189-92.

DOI: dx.doi.org/10.21276/aan.2019.4.2.43 